Fass, R.J., Perkins, R.L. \& Saslaw, S. (1970) Cephalexina new oral cephalosporin: clinical evaluation in sixty-three patients. American Journal of Medical Sciences, 259, 187.

Foord, R.D. (1970) Quoted in Postgraduate Medical Journal, Oct. Suppl. 46, 108.

FooRD, R.D. (1971) Personal communication.

GiRDwOoD, R.H. (1971) The effect of drugs on the blood. In: Symposium on the Adverse Effects of Drugs (Ed. by G. C. Hanson). Beecham Research Laboratories Publication.

Gralnick, H.R., WRIGHT, L.D. \& MCGINNISS, M.H. (1967) Coombs' positive reactions associated with sodium cephalothin therapy. Journal of the American Medical Association, 199, 725.

Kaplan, K., Reisberg, B. \& Weinstein, L. (1968) Cephaloridine. Studies of therapeutic activity and untoward effects. Archives of Internal Medicine, 121, 17.

Kosakai, N. \& Miyakawa, C. (1970) Fundamental studies on the positive Coombs' tests due to cephalosporins. Postgraduate Medical Journal, Oct. Suppl. 46, 107.
Ky, N.T., Chauvin, M.T., Pinon, C. \& Halpern, B.N. (1970) Positive immunological reactions to cephaloridine in patients allergic to penicillin: a study performed with the lymphoblastic transformation test. Postgraduate Medical Journal, Oct. Suppl. 46, 109.

Mine, Y., Nishida, M., Goto, S., Kuwahara, S. (1970) Studies on direct Coombs' reaction by cefazolin in vitro. Journal of Antibiotics, 23, 575.

Molthan, L., Reidenberg, M.M. \& Eichman, M.F. (1967) Positive direct Coombs' tests due to cephalothin. New England Journal of Medicine, 277, 123.

Perkins, R.L., Saslaw, S. \& Billmaier, D. (1967) Coombs' reactivity after cephalothin or cephaloridine. Clinical Research, 15, 426.

Pool, J.G., Hershgold, E.J. \& Pappenhagen, A.R. (1964) High potency antihaemophilic factor prepared from cryoglobulin precipitate. Nature, 203, 312.

YORK, P.S. \& LANDES, R. (1968) Coombs' positive reactions associated with cephaloridine therapy. Journal of the American Medical Association, 206, 1086.

Postgraduate Medical Journal (March 1972) 48, 188-191.

\title{
An unusual case of acrodystrophic neuropathy
}

\author{
R. C. Hilton \\ M.B., M.R.C.P., D.C.H. \\ Manchester Royal Infirmary
}

THIS rare condition is characterized by progressive loss of sensation which usually occurs first and is most severe in the lower limbs, but may eventually become widespread. Thévenard (1953) reviewed 100 cases reported up to that time and since then others have been reported by Ogden, Robert \& Carmichael (1959); Pallis \& Schneeweise (1962); Walker (1955); and Spillane \& Wells (1969). It may occur as a familial disease, when it is often transmitted as an autosomal dominant character but other forms of inheritance can occur (Denny-Brown, 1951).

The familial form is characterized by recurrent, painless ulcers starting on the feet, usually in early adult life, with ultimate severe destruction of the feet and often of the lower legs. Sensation is usually first affected in the territory of one first sacral root, and in the earlier stages there is dissociated sensory loss, temperature sensibility being more extensively and severely impaired than pain, which is in turn lost more than touch. As more adjacent roots become involved this dissociation becomes less marked.

Sporadic cases also occur and it has been suggested that in these there tends to be a more widespread involvement of the sensory roots, an earlier onset of ulceration of the feet and lack of progression of the sensory neuropathy (Ogden, Robert \& Carmichael, 1959). Nerve deafness and lightning pains in the limbs may occur in either form.
The essential pathological change appears to be degeneration of the cells in the posterior root ganglia, and of their fibres both distal and proximal to the ganglia (Denny-Brown, 1951). The anterior roots are not involved; neither does there appear to be any primary involvement of muscle.

The cause is unknown and Denny-Brown (1951) has suggested that this may represent one end of a spectrum of conditions affecting nerve roots, the analogous disease of the anterior roots being the classical radicular form of peroneal muscular atrophy.

The patient here reported, a single woman now aged 60 years, presented with unusually extensive sensory loss and some other features of interest.

\section{Case history}

The details of this patient's early history are vague, but it is known that although her hands and feet appeared normal at birth she did not walk until the age of 3, and then only with difficulty. She later developed ulcers on the plantar and dorsal aspects of the feet, and the feet gradually became deformed, requiring an operation at about the age of 16 years. At a later date bone was discharged spontaneously from both feet. By the age of $\mathbf{3 0}$ she was unable to stand or walk. She has noted shooting pains in the upper limbs and repeated infections of the fingers over many years, and at the age of 50 her right index 
finger was amputated for sepsis; she has since had recurrent indolent fissures on several digits.

When fifty-four, further discharge of bone fragments occurred from ulcers on her feet, and she developed incipient gangrene of the middle three toes of the left foot which later healed, and she has had no further infections of the feet. She has never worked and has been in a home for the disabled for many years.

From early childhood she had frequent episodes of abdominal pain, vomiting and diarrhoea, and because of these she missed practically all her schooling and is unable to read or write. She was admitted to hospital on five or six occasions in such attacks and following a severe attack with intestinal obstruction at the age of 57, underwent repair of a large diaphragmatic hernia containing loops of colon, since when she has had no further abdominal symptoms.

\section{Family history}

Her father died aged 56 of pneumonia but her mother aged 91 is well. Her only sister aged 60 has mild primary generalized osteoarthrosis in the hands and feet but neither she nor her mother had any evidence of a neuropathy when examined.

\section{On examination}

Her general health is good and the viscera are clinically normal, the blood pressure being $160 / 90$ $\mathrm{mmHg}$. She has corneal scarring in the left eye and bilateral early cataracts. The peripheral pulses are of good volume and only the right posterior tibial pulse is impalpable, probably due to deformity of the right root. Oscillometric pulsations in all limbs are normal.

Nervous system. Mentally the patient is severely subnormal with an IQ of 55, but there is no evidence of recent intellectual deterioration.

Cranial nerves: normal apart from impairment of corneal sensation and pin-prick, and light touch sensibilities in the territory of the first divisions of the trigeminal nerves.

Motor functions: muscle tone is normal and the bulk and power of the muscles appear commensurate with her limited activity. The tendon reflexes and the plantar responses are absent.

Sensation: Pin-prick and light touch sensation are absent up to the fourth thoracic segments and over the whole of the upper limbs. Loss of temperature sensation is less marked extending distally from just above the elbows and from the mid thighs. Vibration sense is absent below the clavicles. Sphincter control is normal. She is aware of passive movement of larger joints but with her eyes closed she is unaware of the position in space of a limb which has been passively moved.
Locomotor system. There is gross osteoarthrosis of several finger joints and the right elbow, and to a lesser degree the acromio-clavicular joints. Some digits are absent (Fig. 1). The palmar fascia and skin are thickened limiting extension of the right medius. There has been considerable absorption of the bone of both feet, more so the right, leaving only remnants. The lower ends of the right tibia and fibula have also disappeared (Fig. 2). The knees and hips are normal and she has a mild dorsolumbar scoliosis.

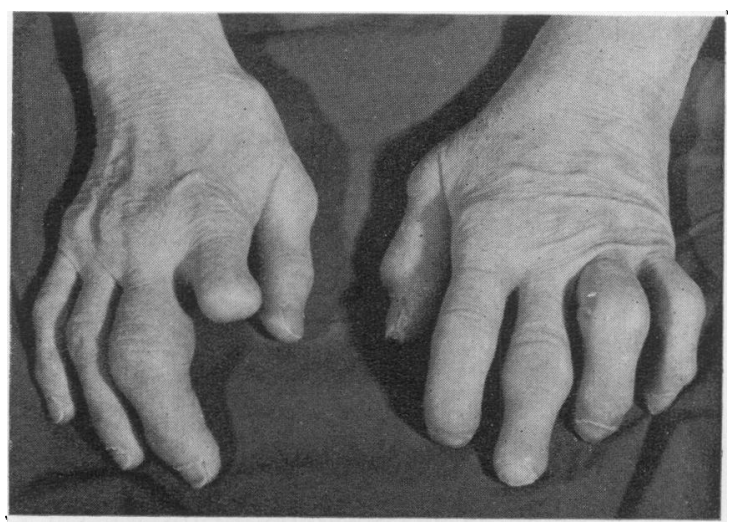

FIG. 1

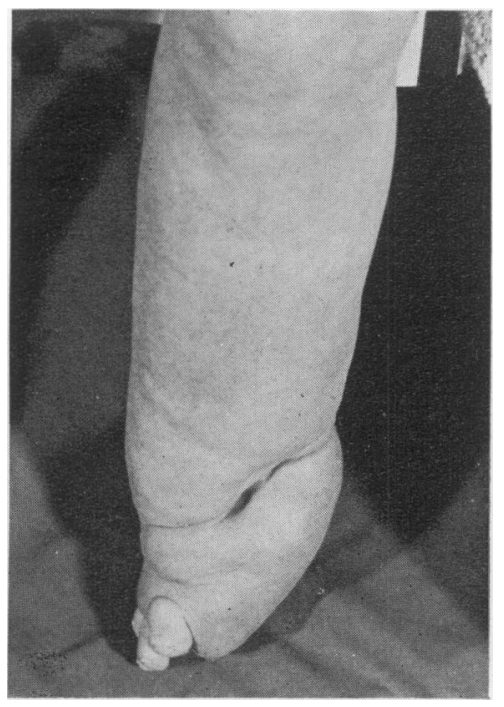

FIG. 2

\section{Investigations}

Electromyography. Left abductor pollicus brevis and left tibialis anterior-no spontaneous activity; complete pattern.

Nerve conduction. Motor fibres: Left median nerve to abductor pollicus brevis; (a) latency at the wrist $3.0 \mathrm{msec}$, and (b) latency at the elbow $7.0 \mathrm{msec}$. 
Sensory fibres: Left index to wrist-no response with $200 \mathrm{~V}$ at $0.1 \mathrm{msec}$. Mixed nerve action potentials: Left median nerve, wrist to elbow-no potentials with $200 \mathrm{~V}$ at $0 \cdot 1 \mathrm{msec}$.

Conclusion: These results are consistent with a sensory neuropathy. There is no evidence of motor nerve involvement.

Cerebrospinal fluid, Protein $26 \mathrm{mg} / 100 \mathrm{ml}$. Serum folic acid, $2 \mu \mathrm{g} / \mathrm{ml}$ (normal above $3 \mu \mathrm{g} / \mathrm{ml}$ ). Serum vitamin $\mathbf{B}_{12}$ and Schilling test, normal. Faecal fats and bromsulphthalein test, normal. Haemoglobin, $11.1 \mathrm{~g} / 100 \mathrm{ml}$; WCC, $8000 / \mathrm{mm}^{3}$. ESR (Westergren), $64 \mathrm{~mm}$; it has remained elevated during 18 months observation in the absence of overt infection. Serum albumen, serum globulin, blood urea and serum calcium and phosphorus were all normal. Wasserman, negative. Urine, sterile. Reactive hyperaemia (right hand), normal. Sweating was detected in the area of impaired sensation after whole body heating and the application of saturated cobalt chloride solution in dehydrated ethanol.

Her visual acuity is $6 / 36$ in each eye and this improves to $6 / 9$ in the right eye and 6/12 in the left eye with correction.

Audiometric testing over a frequency range of $250-4000$ cycles/sec demonstrated in the left ear a maximum loss of 15 decibels at 1000 cycles/sec, and in the right ear a maximum loss of $\mathbf{3 0}$ decibels at 2000 and 4000 cycles/sec.

$X$-rays. Those of the right foot (Fig. 3) show gross absorption and fusion of the lower ends of the tibia and fibula together with absorption of the metatarsals and phalanges, hypoplasia of the tarsal bones and fusion of the tarsal joints. Similar less marked changes are present in the left foot.

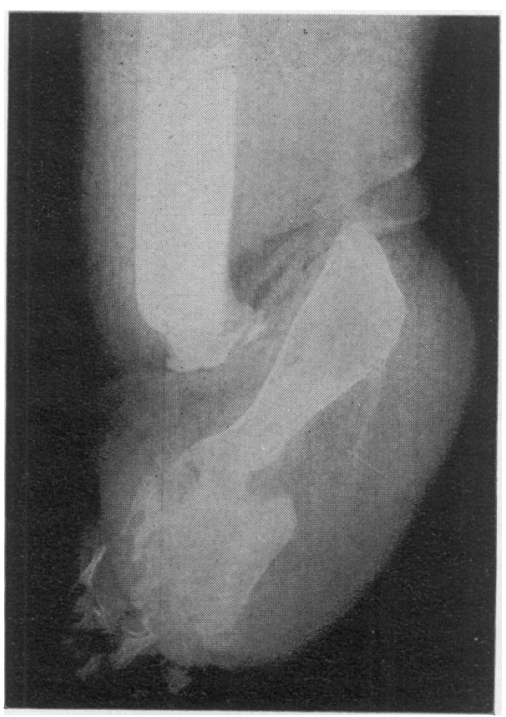

Fig. 3
X-rays of the hands (Fig. 4) show evidence of amputation of the distal two segments of the right index finger and absorption of part of the distal phalynx of the left medius. Osteoarthrotic changes are present in the interphalangeal, metacarpal phalangeal, first carpo-metacarpal and radio carpal joints, with mild juxtra-articular porosis. X-rays of the right elbow show a neuropathic joint with gross new bone proliferation and a large loose body anteriorly whilst in the left elbow there is only mild new bone formation anteriorly and a small loose body.

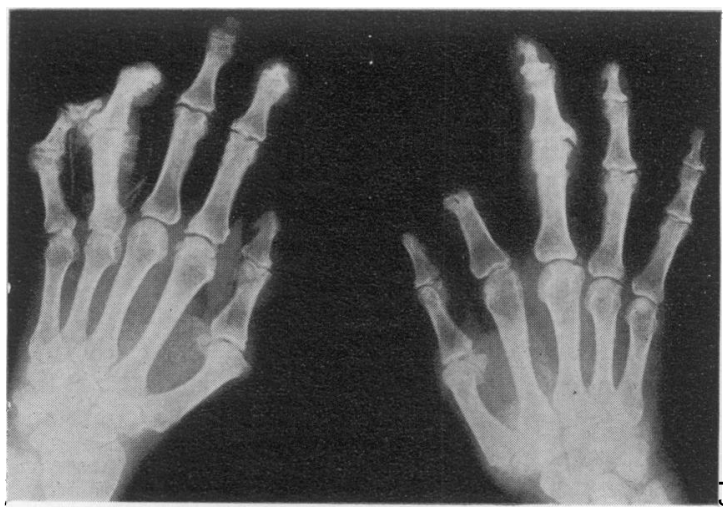

FIG. 4

Pelvic X-rays are normal, apart from small protuberances of new bone from the lesser trochanters of both femora into the psoas tendons. Knee X-rays -normal.

\section{Comment}

There are three features in this patient meriting comment. First, since her sister has primary generalized osteoarthrosis (Kellgren \& Moore, 1952) in a minor form and a familial incidence of this condition has been shown (Lawrence, De Graaff \& Laine, 1962), it seems likely that the striking osteoarthrosis which she has developed in the upper limbs is of the same nature, the severity having been enhanced by an element of neuropathic arthropathy, because she has continued to use the upper limbs in spite of the severe sensory defect in them. Neuropathic arthropathy as shown on the X-ray of the patient's right elbow is unusual in acrodystrophic neuropathy. On the other hand, disuse may have been an important factor in her failure to develop osteoarthrosis in either knee.

Second, three of the eleven affected members of the family reported by Denny-Brown (1951) suffered from recurrent, and a fourth from terminal diarrhoea; barium studies were not reported. In this patient similar symptoms dating from childhood were 
relieved by repair of a large congenital diaphragmatic hernia, so that the possibility of congenital abnormalities of the alimentary tract in other cases should be borne in mind.

Finally, the association of mental subnormality with acrodystrophic neuropathy is unusual and possibly coincidental, although two cases reported by Ogden et al. (1959) had an IQ in the low normal range.

\section{Acknowledgments}

I would wish to thank Dr J. Sharp for his helpful advice, and Mrs M. A. Huddy for carrying out the psychological testing.

\section{References}

DENNY-BRown, D. (1951) Hereditary sensory radicular neuropathy. Journal of Neurology, Neurosurgery and Psychiatry, 14, 237.
Kellgren, J.H. \& Moore, R. (1952) Generalised osteoarthritis and Heberden's nodes. British Medical Journal, $1,181$.

LAWrence, J.S., De GraAFF, F. \& LAINE, V.A.I. (1962) The Epidemiology of Chronic Rheumatism. Blackwell Scientific Publications, Oxford.

Ogden, T.E., Robert, F. \& Carmichael, E.A. (1959) Some sensory syndromes in children: indifference to pain and sensory neuropathy. Journal of Neurology, Neurosurgery and Psychiatry, 22, 267.

Pallis, C. \& Schneeweise, J. (1962) Hereditary sensory radicular neuropathy. American Journal of Medicine, 32, 110.

SpIllane, J.D. \& Wells, C.E.C. (1969) Acrodystrophic Neuropathy. Oxford Medical Publications, London.

ThévenARD, A. (1953) L'acropathie ulcéro mutilante familiale. Acta neurologica et psychiatrica Belgica, 53, 1.

WALKer, C.H.M. (1955) Sensory radicular neuropathy, report on two cases. Great Ormond Street Journal, 10, 72. 